Musées, Patrimoine et Culture scientifiques et techniques

$192 \mid 2020$

novembre-décembre 2020

\title{
Conserver et exposer au centre culturel de Vanuatu
}

Alice Bernadac, Léo Bartoletti et Kaitip Kami

\section{OpenEdition \\ Journals}

Édition électronique

URL : https://journals.openedition.org/ocim/4148

DOI : $10.4000 /$ ocim. 4148

ISSN : 2108-646X

Éditeur

OCIM

Édition imprimée

Date de publication : 1 novembre 2020

Pagination : 16-21

ISSN : 0994-1908

Référence électronique

Alice Bernadac, Léo Bartoletti et Kaitip Kami, «Conserver et exposer au centre culturel de Vanuatu », La Lettre de I'OCIM [En ligne], 192 | 2020, mis en ligne le 01 novembre 2021, consulté le 11 décembre 2021. URL : http://journals.openedition.org/ocim/4148; DOl : https://doi.org/10.4000/ocim.4148

Ce document a été généré automatiquement le 11 décembre 2021.

Tous droits réservés 


\title{
Conserver et exposer au centre culturel de Vanuatu
}

\author{
Alice Bernadac, Léo Bartoletti et Kaitip Kami
}

Figures de grade de l'île d'Ambrym exposées dans le parcours permanent du musée.

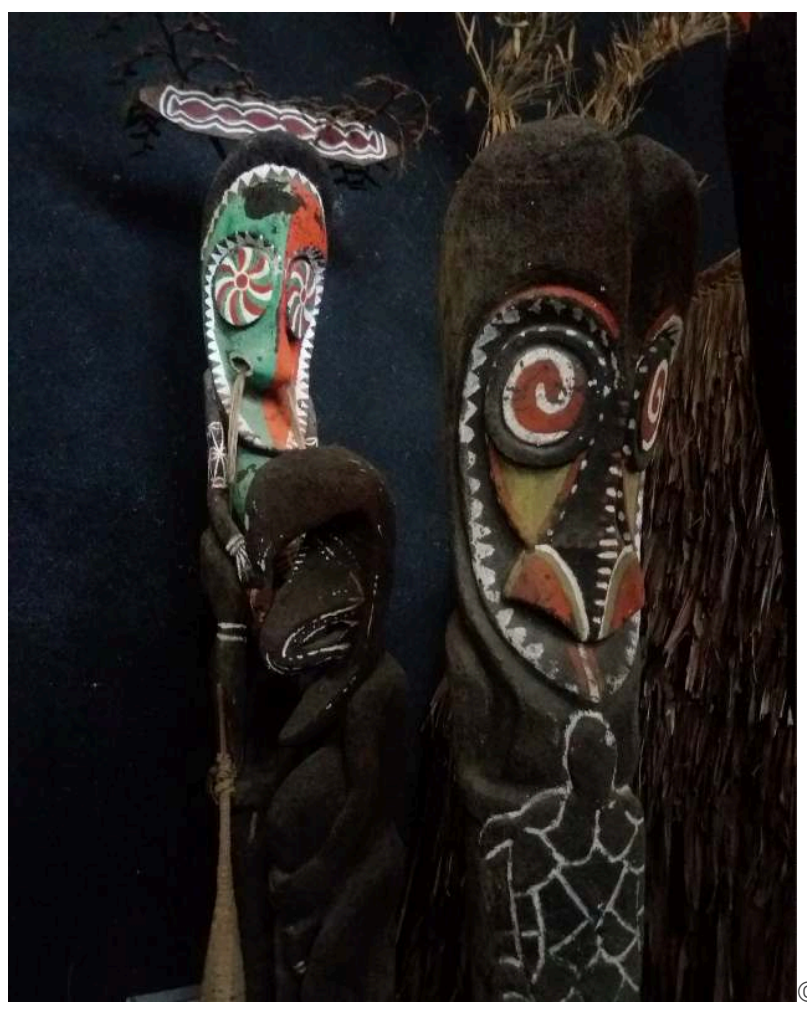

A. Bernadac/Vanuatu Kaljoral Senta

1 Le Centre culturel de Vanuatu, ou Vanuatu Kaljoral Senta (VKS) en bichelamar ${ }^{1}$, aime à se définir comme une "organisation parapluie». Au sein de cette institution composite cohabitent en effet le musée, une bibliothèque et les archives nationales avec une unité dédiée à la préservation des monuments et des sites, un service de photographies et d'enregistrements vidéo et sonores et plusieurs programmes de recherche impliquant 
des communautés de l'ensemble de l'archipel. Ces composantes partagent des locaux deux bâtiments situés face au parlement dans la capitale Port-Vila sur l'île d'Éfaté mais également un but commun: la préservation et la valorisation de l'héritage culturel de l'archipel sous toutes ses formes.

La notion de «kastom» tient une place importante dans la définition de cet héritage et imprègne largement les pratiques du Centre culturel. Le terme kastom désigne en bichelamar l'ensemble des pratiques et modes de vie pré-coloniaux et a joué un rôle central dans les luttes indépendantistes et l'affirmation d'une identité ni-vanuataise ${ }^{2}$. La documentation mais également la préservation et la transmission de la kastom constituent ainsi des missions centrales pour le Centre culturel. Les différentes composantes de l'institution couvrent par conséquent des champs très larges et permettent de traiter à la fois les aspects matériels et immatériels de l'héritage culturel de l'archipel.

Coiffes du sud de l'île de Malekula présentées dans le parcours permanent du musée national de Vanuatu.

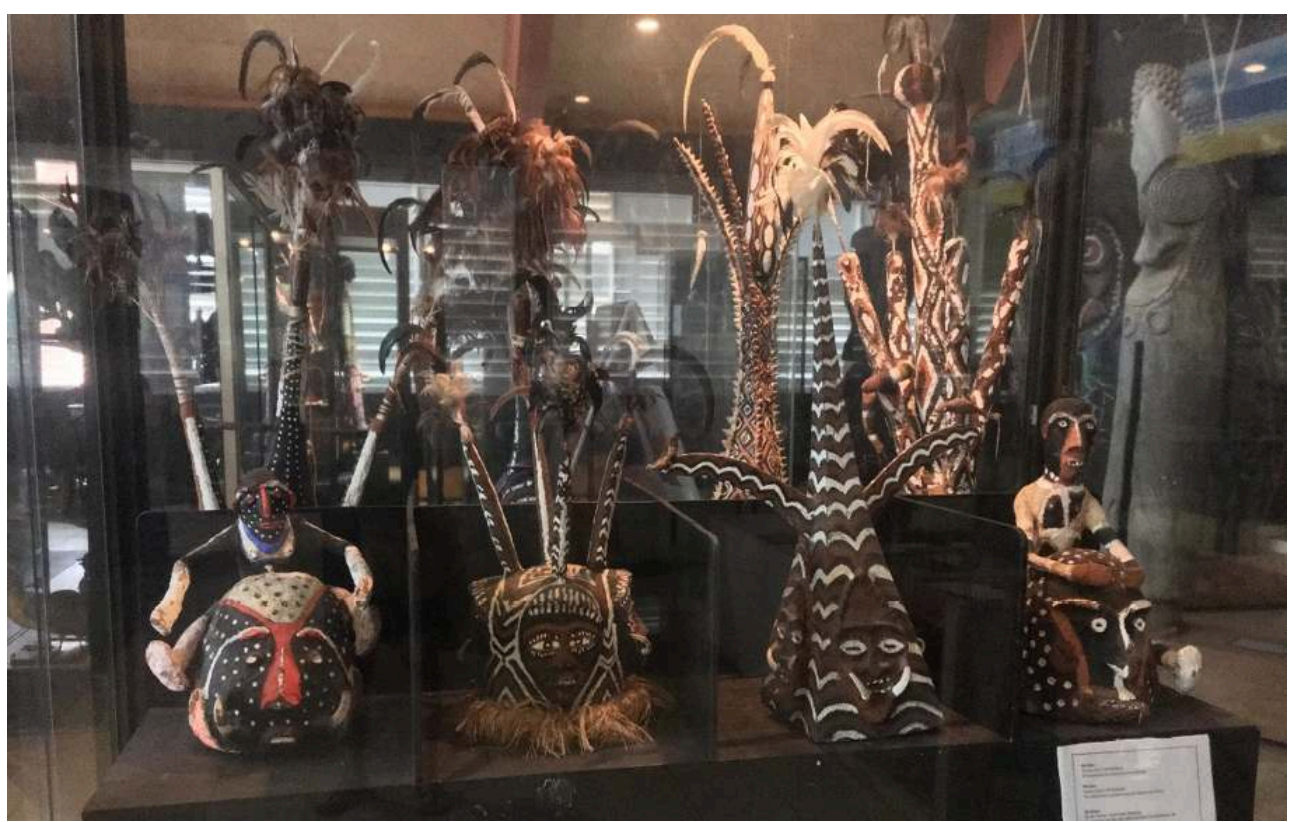

(C) M. Bertin/Vanuatu Kaljoral Senta

Le Vanuatu National Heritage Registry (VNHR) assure par exemple la préservation des sites présentant une importance historique, culturelle ou archéologique. Il gère les fouilles, ainsi que la conservation et l'étude du matériel archéologique. L'Oral Tradition Project ainsi que le Woman Culture Project sont à l'origine de la mise en place d'un vaste réseau de fieldworkers. Ces fieldworkers sont des personnes issues des différentes communautés de l'archipel formées aux techniques de l'ethnographie. Elles assurent, avec le soutien du Centre culturel, la collecte d'artefacts, mais aussi la collecte d'enregistrements et de photographies visant à documenter les pratiques culturelles de leur communauté. Elles opèrent également comme relais du Centre culturel dans les îles et accompagnent les chercheurs. Enregistrements sonores et vidéos réalisés par les fieldworkers ainsi que par la National Film, Sound and Photo Unit rejoignent ensuite la réserve des archives nationales. Le musée a quant à lui la responsabilité d'un espace 
d'exposition et d'une collection d'environ 4000 artefacts et spécimens d'histoire naturelle.

4 Si elles possèdent chacune des missions et un personnel propre, il est très difficile pour ne pas dire impossible - de traiter des différentes entités qui composent le Centre culturel de façon clairement distincte. Il existe en effet très peu de cloisonnements entre les différents services qui collaborent constamment les uns avec les autres. Le musée national ne fait pas exception. La gestion de ses collections met ainsi en œuvre des principes communs avec les archives nationales, fondés sur le respect de la kastom. Bien que le musée conserve des artefacts, leur gestion est également profondément influencée par la place importante accordée à la dimension immatérielle du patrimoine au sein des autres services du Centre culturel. La salle d'exposition du musée apparaît quant à elle comme un espace polyphonique où ne s'exprime pas seulement la vision du conservateur, mais bien celle de l'ensemble des entités qui composent l'institution.

\section{Gérer les collections dans le respect de la kastom}

5 Les collections du musée national couvrent l'ensemble de l'histoire du Vanuatu. Quelques pièces archéologiques sont toujours gérées par le musée auxquelles il faut ajouter des œuvres contemporaines et des collections liées à l'histoire récente du pays, principalement à l'Indépendance et à la Seconde Guerre mondiale. La majorité de la collection est composée d'objets liés à la kastom: tambours à fente, instruments de musique, nattes, coiffes...

Certains des objets conservés par le musée sont l'héritage de la période coloniale. Le Centre culturel a en effet été créé en 1956 à l'époque où l'archipel était un condominium franco-britannique. Ce premier centre était déjà composite puisqu'il rassemblait une bibliothèque et un musée. Le musée actuel conserve également de nombreux objets plus récents issus de collectes ou déposés par les communautés via le réseau des fieldworkers. Certaines régions sont mieux représentées dans les collections que d'autres, en fonction des études dont elles ont fait l'objet mais également de la volonté des communautés de voir - ou non - leur patrimoine intégrer le musée.

7 En effet, nombre des objets conservés au Centre culturel sont soumis à un ensemble complexe de droits qui régissent leur fabrication, leur utilisation, mais également leur reproduction photographique et leur éventuelle exposition à un public extérieur. En fonction des communautés, des restrictions peuvent s'appliquer selon l'âge, le genre ou le degré d'initiation. L'acquisition du droit de fabriquer ou d'utiliser un objet peut notamment faire l'objet d'un paiement. Il n'est donc pas évident pour les communautés d'accepter que certains artefacts puissent rejoindre les collections du musée et encore moins qu'ils puissent être montrés au public. Par ailleurs, les objets qui composent les collections du Centre culturel demeurent soumis à ces systèmes de droits et des restrictions s'y appliquent sous le contrôle de leur communauté d'origine ${ }^{3}$.

Le musée est ainsi bien plus dépositaire, ou "gardien » d'une partie de ses collections ${ }^{4}$. Il en assure certes la préservation mais se doit aussi d'agir systématiquement en accord avec les communautés-sources. Le réseau des fieldworkers joue un rôle fondamental dans le maintien des relations avec les ayants-droits des objets. De ce système découlent certaines particularités dans la façon de gérer et de conserver les collections. Tout d'abord, une distinction est établie entre les objets relevant de la kastom masculine 
et de la kastom féminine. Cette distinction prend notamment la forme d'une séparation spatiale dans deux réserves différentes. La coordinatrice du Woman's Culture Project prend également en charge, aux côtés du conservateur, la gestion des objets liés à la kastom des femmes.

La mezzanine de l'espace d'exposition permanente.

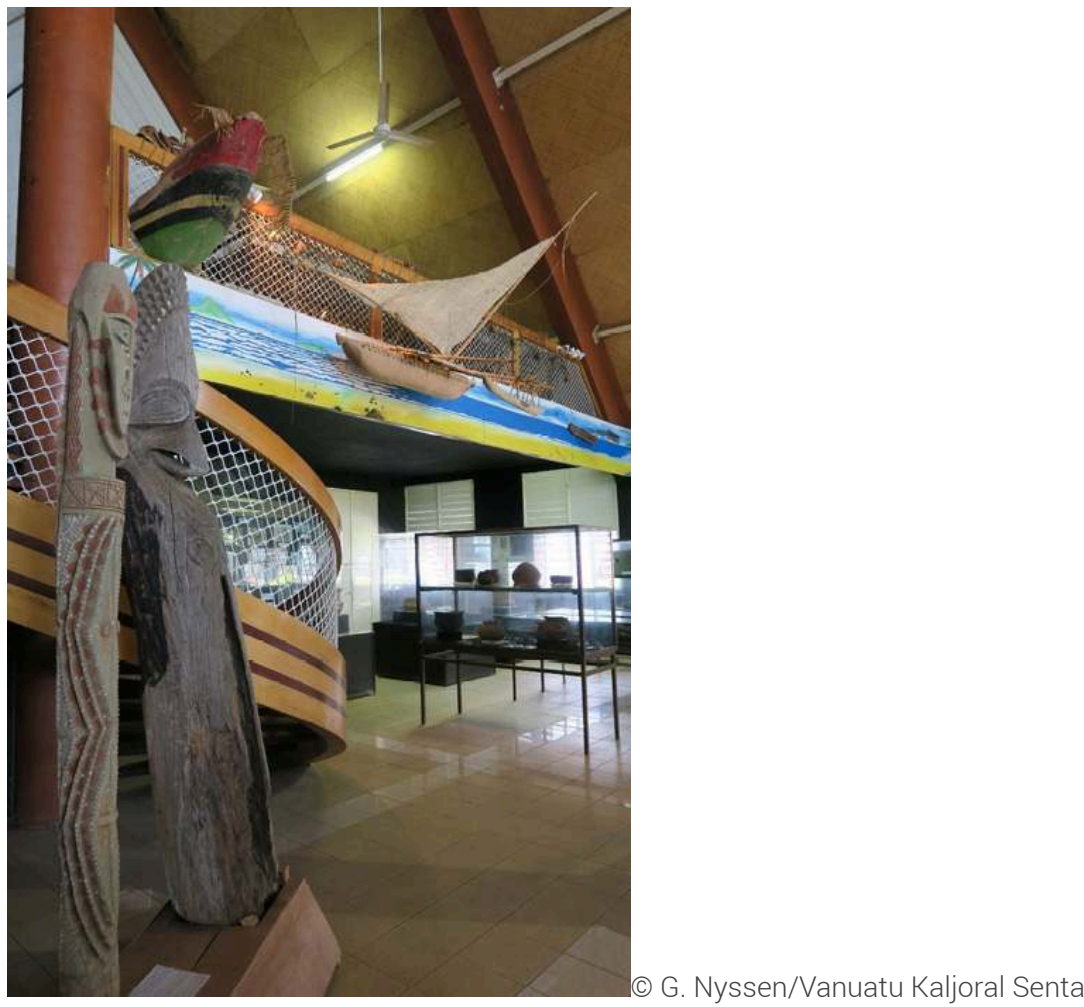

9 Par ailleurs, l'exposition et la reproduction photographique des objets sont soumises à l'autorisation des ayants-droits. Certains objets conservés dans les réserves du musée n'ont ainsi pas vocation à être exposés publiquement mais seulement à être préservés. En dehors du personnel du musée, ils ne sont accessibles qu'aux membres autorisés de leur communauté d'origine.

Ce système a également des conséquences sur la restauration des objets. En effet, la fabrication de certaines typologies étant soumise à des droits, il n'est pas possible pour une personne ne possédant par ces droits d'intervenir sur ces objets. C'est notamment le cas des coiffes en fougère arborescente produites dans le sud de l'île de Malekula, nombreuses dans les collections du musée. Étant conçues pour un usage éphémère, ces objets représentent un défi pour ce qui est de leur conservation, d'autant qu'ils sont toujours susceptibles d'être réutilisés dans le cadre de performances.

11 Car les objets conservés au Centre culturel n'ont pas vocation à demeurer sur leurs étagères ou dans leurs vitrines. Partie intégrante de la kastom, ils contribuent à la faire connaître par le biais du musée, mais également à la faire vivre en continuant d'être utilisés lors de certaines occasions comme le Vanuatu National Arts Festival. Cette nécessité de maintenir les objets «vivants » peut parfois primer sur leur conservation matérielle ${ }^{5}$. Couplés aux archives sonores et visuelles, ils servent également de ressources mises à disposition des communautés pour transmettre ou se réapproprier certaines pratiques. 
Bien qu'elles soient gérées par des entités différentes, les collections du musée et des archives peuvent par ailleurs être considérées comme un tout. Les collections des archives sont soumises aux mêmes restrictions que celles du musée et répondent aux artefacts que celui-ci conserve. La création d'une base de données commune est d'ailleurs prévue de longue date ${ }^{6}$. Les collections des archives ne sont cependant pas tant envisagées sous l'angle de la documentation des objets que comme une kastom bank, une banque coutumière, valant pour elle-même ${ }^{7}$.

\section{Un parcours d'exposition à plusieurs voix}

13 Le Centre culturel dispose d'un espace d'exposition ouvert au public composé d'une grande salle et d'une mezzanine accessible par un escalier. L'espace est réparti entre une salle d'exposition permanente, une salle d'exposition temporaire d'une vingtaine de $\mathrm{m}^{2}$ et une boutique souvenirs. Bien que sa gestion lui revienne en grande partie, cet espace n'est pas uniquement investi par le musée mais bien par l'ensemble des acteurs qui composent le Centre culturel. Son organisation fait l'objet d'une médiation entre ces différents acteurs et leurs visions des missions du centre et de ses collections. Le VNHR dispose ainsi d'un espace dédié au sein du parcours permanent qui lui permet d'exposer les collections archéologiques dont il a la charge.

14 L'entrée du musée est consacrée aux pratiques immatérielles. Elle constitue à la fois un espace d'accueil du public, d'enseignement et de convivialité. Des bancs y encadrent un bac rempli d'une fine couche de sable ainsi que plusieurs instruments de musique. C'est ici que débutent les visites guidées par une démonstration de sand drawing réalisée par le guide du musée. La pratique du dessin sur sable est commune à plusieurs régions du Vanuatu. Ces dessins, tracés en une seule ligne continue, peuvent intervenir dans plusieurs contextes: jeux, messages, rituels... Leur réalisation s'accompagne de la récitation de l'histoire associée au dessin. Depuis 2008, le sand drawing est inscrit sur la liste du patrimoine immatériel de l'humanité par l'Unesco. 
La salle d'exposition, " un espace polyphonique où ne s'exprime pas uniquement la vision d'un conservateur de musée. "

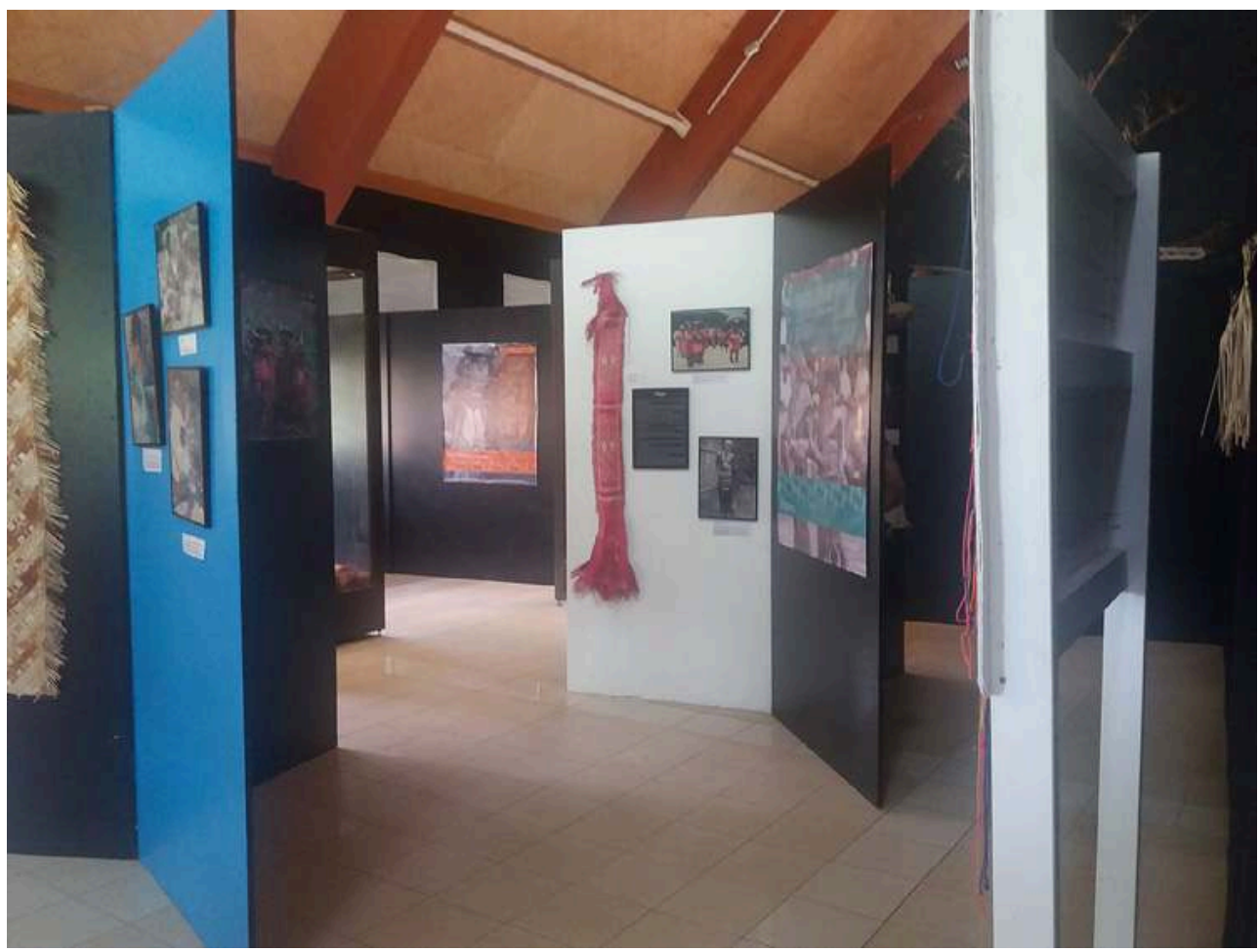

(c) A. Bernadac/Vanuatu Kaljoral Senta

15 La présence au sein du parcours d'exposition d'un espace dédié à cette pratique souligne une fois encore l'importance accordée au sein du Centre culturel aux dimensions immatérielles de la kastom. Il ne s'agit pas uniquement de présenter le sand drawing au public, mais également d'assurer sa transmission. L'entrée du musée est en effet un lieu de rencontre et de réunion informelle. Enfants, acteurs internes et externes au Centre culturel s'y retrouvent régulièrement l'après-midi pour visionner des documentaires, pour s'abriter de la pluie ou tout simplement pour discuter. À ces occasions, chacun est libre de s'emparer du bac de sand drawing et de s'exercer ou d'apprendre de nouveaux dessins aux plus jeunes. Les personnes présentes commentent, corrigent ou encouragent les essais et échangent autour des histoires liées au dessin ou sur d'autres sujets. Depuis le printemps 2020, l'entrée du musée accueille également la kastom skul, l'école coutumière animée par le Centre culturel qui constitue un espace de transmission plus formalisé des pratiques coutumières.

\section{Projet de nouveau parcours permanent}

La salle d'exposition du Centre culturel est donc avant tout un espace d'échanges entre des acteurs divers et non pas uniquement un espace dédié à l'exposition de la culture matérielle de l'archipel. C'est cette vision qui présidait au projet de renouvellement du parcours permanent. Ce projet, dont l'avenir a été rendu incertain par la crise sanitaire liée au Covid-19, s'inscrivait dans le cadre de la célébration des 40 ans de l'indépendance du Vanuatu organisée par le Centre culturel et qui aurait dû avoir lieu en juillet 2020. Il s'agissait pour le Centre culturel d'élaborer un nouveau parcours de visite qui réponde aux ambitions de l'institution vis-à-vis de son public et à ses missions 
de valorisation et de transmission du patrimoine de l'archipel. Le parcours actuel présente en effet un certain nombre de problèmes aux yeux de l'équipe du centre. L'espace d'exposition étant un vaste quadrilatère, il est difficile d'y délimiter un parcours et un sens de visite. Seul l'espace d'exposition temporaire est clairement encadré par des cloisons. Cette configuration pose aujourd'hui des soucis de lisibilité du parcours par les visiteurs. Ce nouveau projet était donc avant tout guidé par une démarche pédagogique.

Encadré par le musée national, le projet impliquait des responsables issus de l'ensemble des services du Centre culturel. Le parcours retenu visait précisément à offrir une place à chacun de ces acteurs et prolongeait l'idée d'un espace d'exposition à plusieurs voix. Un découpage en différentes sections, chacune confiée à une équipe différente, devait permettre de clarifier le parcours de visite et lui donner un sens logique en le faisant débuter par l'archéologie, actuellement située au fond de la salle d'exposition. Les deux sections suivantes devaient présenter successivement la kastom masculine et la kastom féminine, ce second espace étant sous la responsabilité du Woman's Culture Project. La mezzanine aurait par ailleurs permis l'aménagement d'une salle intitulée Spirits World, abordant certains aspects rituels plus secrets. L'idée était de profiter de l'isolement de la mezzanine pour permettre au public qui ne le souhaiterait pas de ne pas être confronté aux objets présentés dans cette partie. Le respect de la kastom demeurait donc une préoccupation importante dans la conception de ce parcours. Une dernière section, abondamment alimentée par les archives, aurait abordé l'histoire du Vanuatu, notamment l'indépendance de l'archipel actuellement présentée en tout début de parcours. La visite se serait achevée au niveau de la boutique de souvenirs et de l'espace d'exposition temporaire. Le projet conservait bien sûr l'espace d'entrée dédié au sand drawing, aux instruments de musique et à la discussion.

\section{Et demain...}

S'il est difficile d'affirmer aujourd'hui quel sera son avenir, ce nouveau parcours s'inscrivait pleinement dans la vision portée par le Centre culturel en proposant un espace polyphonique où ne s'exprime pas uniquement la vision d'un conservateur de musée. Dans ce concert de voix, le musée apparaissait avant tout comme un coordinateur. Il était également porteur d'un discours plus technique concernant la conservation matérielle des objets et leurs conditions d'exposition avec une attention portée aux dispositifs muséographiques (principalement vitrines et éclairage). Ce discours est sans doute ce qui fait la spécificité du musée au sein d'une institution moins centrée sur la dimension matérielle des objets que sur leur capacité à continuer à générer des relations ${ }^{8}$. Au-delà des objets, le parcours permanent du musée incarne luimême cette capacité. Le parcours de 1995, réalisé à l'occasion de l'installation du Centre culturel dans ses locaux actuels, avait ainsi été conçu en très étroite collaboration avec les fieldworkers, symbolisant les liens continus du Centre avec les communautés de l'archipel. Comme un écho, le nouveau projet de parcours illustrait cette fois sa capacité à générer et à stimuler les relations existantes entre les différentes parties du Centre culturel et leurs différentes visions du patrimoine ni-vanuatais. 


\section{BIBLIOGRAPHIE}

Regenvanu R. The Vanuatu Cultural Centre efforts towards protecting indigenous intellectual property rights in Vanuatu. Communication, Indigenous People's Knowledge \& Intellectual Property Rights Consultation (Suva, Fiji du 24 au 27 Avril 1995), 210 p.

Stanley N. The future of indigenous museums : perspectives from the Southwest Pacific. New York, Oxford : Berghahn Books, 2007.

Centre culturel de Vanuatu (présentation du Centre culturel et du musée national ; obtention de permis de recherche ; programmation) : https://vanuatuculturalcentre.gov.vu

\section{NOTES}

1. Le bichelamar est la langue véhiculaire du Vanuatu, l'une des trois langues officielles avec l'anglais et le français.

2. Alivizatou M. At the inferface of kastom and development: the case of the Vanuatu Cultural Centre. In Alivizatou M. Intangible heritage and the museum : new perspectives on cultural preservation. Walnut Creek : Left Coast Press Inc., 2012, pp. 77-104.

3. Bertin M. Allier kastom et tabu au musée : gestion et exposition des objets du Vanuatu, Icofom Study Series, vol. 47, 2019, pp. 41-56.

4. Geismar H. Cultural property, museums, and the Pacific : reframing the debates, International Journal of Cultural Property, 2008, pp. 109-121.

5. Alivizatou M. op. cit. pp. 77-104.

6. Mohns W. The digital archive and catalogues of the Vanuatu Cultural Centre: overview collaboration and future directions. In Taylor J. et Thieberger N. Working together in Vanuatu: research histories, projects and reflections. Canberra: Australia National University, 2011, pp. 141-150.

7. Kapere J. The Vanuatu National Film Unit. In Taylor J. et Thieberger N. op. cit. pp. 133-140.

8. Geismar H. et Tilley C. Negociating materiality: international and local museum practices at the Vanuatu Cultural Centre and National Museum, Oceania, nº73, 2003, pp. 170-188.

\section{RÉSUMÉS}

Le Centre culturel de Vanuatu veille à la préservation et à la transmission de la culture traditionnelle de l'archipel : la kastom. Le savoir se partage entre fieldworkers et conservateurs pour gérer, exposer et constituer leur patrimoine matériel et immatériel.

\section{INDEX}

Mots-clés : dispositif participatif, centre d'interprétation, patrimoine 


\section{AUTEURS}

\section{ALICE BERNADAC}

Conservatrice de la Cité internationale de la Tapisserie d'Aubusson

bernadac.alice@gmail.com

\section{LÉO BARTOLETTI}

Assistant muséographe au musée national de Vanuatu à Port-Vila leo.bartoletti1997@gmail.com

\section{KAITIP KAMI}

Conservateur au musée national de Vanuatu à Port-Vila

kkami@vanuatu.gov.vu 